\title{
GERMINAÇÃo DE SEMENTES DE PLANTAS MEDICINAIS LENHOSAS
}

\author{
Shirley G.T.da Rosa ${ }^{1}$ \\ Alfredo Gui Ferreira ${ }^{2}$
}

Recebido em 10/08/00. Aceito em 26/04/01

\begin{abstract}
RESUMO - (Germinação de sementes de plantas medicinais lenhosas) A germinação de sementes de Aloysia gratissima, Bauhinia forficata; Casearia sylvestris, Dodonea viscosa, Psychotria leiocarpa e Sida rhombifolia foi verificada usando-se agar a $1 \%$ em placas de petri ou areia como substrato. Foi testada a germinação em temperaturas constantes de $20,25,30$ e $35^{\circ} \mathrm{C}$ na luz ou no escuro, além de 10 e $15^{\circ} \mathrm{C}$ somente na ausência de luz. Foram também usadas temperaturas diárias alternantes de $10-20^{\circ} \mathrm{C} ; 15-25^{\circ} \mathrm{C}, 20-30^{\circ} \mathrm{C}$ e $25-35^{\circ} \mathrm{C}$ sendo por 9 horas na temperatura mais alta na luz, com intensidade de radiação entre 40 e $60 \mu \mathrm{mol} \cdot \mathrm{m}^{-2} \cdot \mathrm{s}^{-1}$. Apenas A. gratissima mostrou-se fotoblástica positiva, porém respondendo melhor à germinação com alternância de temperaturas. Em C. sylvestris e P. leiocarpa em temperaturas constantes só ocorreu germinação a 20 ou $25^{\circ} \mathrm{C}$. Sementes de B. forficata germinaram acima de $80 \%$ entre 15 e $30^{\circ} \mathrm{C}$ constantes ou quando uma das temperaturas do par alternante estivesse entre estas duas temperaturas. D. viscosa, após escarificação das sementes, mostrou ampla faixa de germinação em diferentes temperaturas ou regime lúmico. S. rhombifolia germinou em taxas mais altas quando a temperatura constante foi $35^{\circ} \mathrm{C}$ ou quando, na alternância, esta temperatura estava presente. Nos tratamentos onde houve mais germinação, o ótimo situou-se em $44 \%$ para $A$. gratissima e $97 \%$ em B. forficata. Em se tratando de espécies nativas sem melhoramento ou seleção de matrizes, estes índices são bastante aceitáveis.
\end{abstract}

Palavras-chave-Aloysia, Bauhinia; Casearia, Dodonea, Psychotria, Sida.

\begin{abstract}
Seed germination of medicinal woody plants)
Seed germination of Aloysia gratissima, Bauhinia forficata; Casearia sylvestris, Dodonea viscosa, Psychotria leiocarpa Sida rhombifolia was verified using agar at $1 \%$ in petri dishes or sand, as substrate. The germination was tested under constant temperatures of $20^{\circ} \mathrm{C}, 25^{\circ} \mathrm{C}, 30^{\circ} \mathrm{C}$ and $35^{\circ} \mathrm{C}$ in light or darkness, and also at $10^{\circ} \mathrm{C}$ and $15^{\circ} \mathrm{C}$ in the dark. Morever, diary alternating temperatures of $10-20^{\circ} \mathrm{C} ; 15-25^{\circ} \mathrm{C} ; 20-30^{\circ} \mathrm{C}$ and $25-35^{\circ} \mathrm{C}$ were used, the higher temperature being in light at a radiating intensity of 40 to $60 \mu \mathrm{mol} \cdot \mathrm{m}^{-2} \cdot \mathrm{s}^{-1}$. Only A. gratissima showed positive photoblastic behavior, but higher germination was found at alternating temperatures. Both, $C$. sylvestris and $P$. leiocarpa at constant temperatures germinated only at 20 or $25^{\circ} \mathrm{C}$. B. forficata seeds germinated more than $80 \%$ from $15^{\circ} \mathrm{C}$ to $30^{\circ} \mathrm{C}$ constant or when one of the shift temperatures was on that range. Scarified seeds of D. viscosa germinated at the same level from $10^{\circ} \mathrm{C}$ to $35^{\circ} \mathrm{C}$. S. rhombifolia seeds germinated in higher rates at the constant temperature of $35^{\circ} \mathrm{C}$ or the alternating ones that included $35^{\circ} \mathrm{C}$ in the treatment. The higher percentage of germination achieved was from $44 \%$ in A. gratissima to $97 \%$ in B. forficata seeds. Since those are native species about which there are no reports on genetic improvement or landrace selection, the percentages of germination obtained were considered acceptable.
\end{abstract}

Key words - Aloysia; Bauhinia; Casearia, Dodonea; Psychotria, Sida.

\footnotetext{
${ }^{1}$ Universidade Federal do Rio Grande do Sul (UFRGS).

${ }^{2}$ Av. Bento Gonçalves 9500, Prédio 43423. Departamento de Botânica, IB, UFRGS, Campus do Vale. CEP 91501-900 - Porto Alegre, RS. Brasil.
} 


\section{Introdução}

A exploração de plantas nativas na medicina popular, é largamente difundida no País. A maioria das espécies tem sido usada de forma extrativista e o crescimento da população humana e a ocupação de áreas naturais vêm aumentando a pressão destrutiva sobre esta flora. A disponibilização de informações sobre a propagação de espécies medicinais, vai facilitar aos agricultores o cultivo destas plantas, favorecendo o mercado informal de erveiros e os pequenos e médios laboratórios farmacêuticos nacionais dedicados à produção de medicamentos de origem vegetal (Farias, 1999). O progresso da fitoterapia e a obtenção de fitofármacos depende do acesso facilitado a plantas produtoras e substâncias ativas, evitando falsificações ou substituição por outras plantas, aumentando a credibilidade nos mesmos por parte de pacientes e médicos (Rosa, 2000).

A propagação de plantas fanerogâmicas medicinais pode ser efetuada por via assexuada, em alguns casos, ou sexuada, esta representada pela germinação de sementes.

A luz e a temperatura são os fatores ambientais de maior importância na promoção da germinação de sementes. Fornecidas as condições ideais de luz e umidade, a temperatura do solo irá determinar quantas sementes germinarão em uma amostra, bem como a velocidade dessa germinação (Heydecker, 1977).

Percepção, interpretação e transdução dos sinais luminosos são captados por fotoreceptores, sendo o fitocromo o principal (Kendrick \& Kronenberg, 1994). Em muitas espécies, a germinação das sementes é mediada pelo fitocromo diante das condições de luminosidade presentes (Scopel et al., 1994; Deregibus et al., 1994). A resposta à luz pode manifestar-se por um incremento na germinação (fotoblastismo positivo) enquan- to, para outras espécies, a ausência de luz é o que promove a germinação (fotoblastismo negativo).

A temperatura influi no processo de germinação, especialmente, por alterar a velocidade de absorção de água e modificar a velocidade das reações químicas que irão acionar o desdobramento, o transporte das reservas e a ressíntese de substâncias para a plântula (Bewley \& Black, 1994). A faixa de temperatura ótima é aquela onde acontece a germinabilidade máxima, registrando-se o percentual mais alto de germinação, no menor tempo médio (Labouriau, 1983).

Sementes de muitas espécies têm germinabilidade mais alta em temperaturas alternantes do que em temperaturas constantes (Toole, 1973; Thompson \& Grime, 1983).

Existem referências de alguns trabalhos sobre a germinação de plantas medicinais do Rio Grande do Sul ( Rosa \& Ferreira, 1998, 1999; Rosa \& Barros, 1999) tendo-se, no presente estudo, examinado a germinação de seis espécies de lenhosas quais foram: 1- Aloysia gratissima - erva-de-nossa-senhora; erva-dagraça, um arbusto de até 3 metros de altura, muito ramificado e espinescente nos ápices. As folhas e os ramos são usados para preparo de expectorante, digestivo e para doenças da bexiga (Alice et al., 1995). 2- Bauhinia forficata - pata-de-vaca, unha-de-vaca; é árvore decídua ou semi-decídua de 5 a 9 metros, característica da Floresta Pluvial Atlântica (Lorenzi, 1992), sendo de suas folhas extraídos princípios diuréticos e hipoglicemiantes (Alice et al., 1995) 3- Casearia sylvestris chá-de-bugre, cafezeiro-do-mato, guaçatunga; é árvore de porte médio, heliófila, com frutos e sementes que são dispersos por passáros (Sanchotene, 1989). As folhas possuem princípios ativos antidiarréicos, antireumáticos, diuréticos e antiinflamatórios (Simões et al., 1986; Lopes, 1997). 4Dodonea viscosa -vassoura-vermelha; vassoura-do-campo; erva-de-veado; é utilizada 
como anti-febril e antirreumática (Simões et al., 1986), sendo arbusto alto (3 a 8 metros), heliófilo, fruto samaróide com três sementes com alas e dispersão anemocórica (Burrows, 1995). 5-Psychotria leiocarpa, arbusto de até 2 metros de altura, frutos drupáceos de coloração azul-violáceo quando maduro (Dillenburg, 1978), com dispersão zoocórica (Reis et al., 1998). Possui atividade analgésica (Elisabetsky et al., 1997). 6- Sida rhombifolia - guanxuma ou guaxuma;- subarbusto de raizes profundas, usadas em infusão para combater o colesterol, a hipertensão , além de ser diurética (Lopes, 1997). Também pode ter ação antiinflamatória e hepatoprotetora (Rao \& Mishra, 1997).

Esse trabalho foi delineado para se verificar a influência das temperaturas, constantes ou alternadas, combinadas à presença ou ausência de luz, na germinação de seis espécies medicinais lenhosas.

\section{Material e métodos}

Frutos ou sementes de Aloysia gratissima (Gill. et Hook) Troncoso (Verbenaceae); de Bauhinia forficata Linck (Caesalpinaceae); de Casearia sylvestris Swartz (Flacourtiaceae); de Dodonea viscosa (L.) Jacquin (Sapindaceae); de Psychotria leiocarpa Cham. et Schlecht. (Rubiaceae) e de Sida rhombifolia L. (Malvaceae), foram colhidos em torno de Porto Alegre $\left(30^{\circ} 02^{\prime} \mathrm{S}, 51^{\circ} 14^{\prime}\right.$ W), RS em 1997 e 1998 (detalhes de coleta em Rosa, 2000).

Após o beneficiamento foi analisado o teor de umidade $\left(105^{\circ} \mathrm{C} \pm 2\right.$ por 24 horas $)$ de no mínimo 20 sementes com duas repetições e o peso de mil sementes (Brasil, 1993).

Para os testes de germinação, desinfestaram-se as sementes recém coletadas, com hipoclorito de sódio a $1 \%$ de cloro ativo por 10 minutos seguido de enxágüe em água estéril. As sementes de D. viscosa foram escarificadas com ácido sulfúri- co $80 \%$ por 4 minutos. As semeaduras foram efetuadas em condições assépticas. Como substrato foram utilizados $20 \mathrm{ml}$ de agar a $1 \%$ em placas de petri de $9 \mathrm{~cm}$ de diâmetro. Também foi usada areia esterilizada umedecida com água destilada como substrato em caixas gerbox. Para estes testes, foi utilizada a resposta no tratamento com agar em que houve maior germinabilidade. Como foi usado o mesmo lote de sementes dos experimentos anteriores, houve armazenagem no ambiente de laboratório $\left( \pm 25^{\circ} \mathrm{C}\right)$ por um período de 30 a 50 dias. A semeadura, em areia, foi feita a uma profundidade aproximada de $1 \mathrm{~cm}$ da superfície, com 8 repetições de 25 sementes.

Os testes de germinação foram realizados em câmaras BOD, com temperaturas constantes de $10^{\circ} \mathrm{C}$ e $15^{\circ} \mathrm{C}$ (na ausência de luz), $20^{\circ} \mathrm{C}, 25^{\circ} \mathrm{C}, 30^{\circ} \mathrm{C}$ e $35^{\circ} \mathrm{C}$ (na ausência e presença de luz) com fluxo de radiação de cerca de $50 \mu \mathrm{mol} \mathrm{m} \mathrm{m}^{-2} \mathrm{~s}^{-1}$ e a ausência de luz foi obtida pelo envolvimento das placas de petri ou caixas gerbox em duas folhas de papel aluminizado. Nestes tratamentos a germinação era verificada sob luz verde de segurança (Labouriau \& Costa, 1976) a cada três dias. Também foi avaliada a germinação em temperatura alternada de $10-20^{\circ} \mathrm{C} ; 15-25^{\circ} \mathrm{C}$; $20-30^{\circ} \mathrm{C} ; 25-35^{\circ} \mathrm{C}$, sendo a temperatura mais alta por nove horas na luz, experimentos acompanhados por 30 a 45 dias.

$\mathrm{O}$ delineamento experimental foi completamente casualisado com quatro repetições de 25 sementes por tratamento. Para análise os dados foram transformados em arc.sen $\sqrt{x}$, submetido a análise de variância (ANAVA) geral, com analise de interação entre luminosidade e temperatura, seguido de teste de Tukey (Sokal \& Rohlf, 1981). O tempo médio de germinação foi calculado de acordo com Ferreira (1977) e o critério de germinação, em agar, foi a emissão da radícula (no mínimo a metade do comprimento da se- 
mente). As sementes que não germinaram foram submetidas ao teste de tetrazólio (Cloreto de trifenil tetrazólio a $1 \%$ em solução aquosa), de 30 minutos a três horas a $35^{\circ} \mathrm{C}$, o qual avalia a atividade de enzimas do grupo das desidrogenases (Grabe, 1970). $\mathrm{Na}$ areia , a emergência da plântula foi o critério de germinação.

\section{Resultados}

As sementes eram pequenas, leves e possuíam menos de $10 \%$ de umidade relativa (Tabela 1), uma das características de sementes ortodoxas. A exceção foi $B$. forficata que apresentou sementes mais pesadas do que as outras espécies e com maior conteúdo de água.

Apenas A. gratissima revelou possuir sementes fotoblásticas positivas (Tabela 2); temperaturas alternantes promoveram a germinação para níveis pouco acima de $30 \%$ (Tabela 3). Nas demais temperaturas, a presença de luz ou ausência de luz foi indiferente (Tabela 3), pelo que foram classificadas como fotoblásticas neutras.

B. forficata teve uma alta germinabilidade (+ $80 \%)$, tanto no escuro como na luz em temperaturas constantes ou alternadas entre $15 \mathrm{e}$ $30^{\circ} \mathrm{C}$. As sementes não germinaram quando a temperatura foi de $10^{\circ} \mathrm{C}$. Casearia sylvestris e Psychotria leiocarpa são duas espécies que apresentaram um comportamento germinativo muito semelhante (Tabela 3) alcançando maiores taxas de germinação em temperaturas constantes de $20^{\circ}$ ou $25^{\circ} \mathrm{C}$. Acima ou abaixo destas temperaturas a germinação foi bastante reduzida. Em temperaturas alternadas para $C$. sylvestris a taxa germinativa foi semelhante àquelas obtidas em temperaturas constantes, à exceção de 25$35^{\circ} \mathrm{C}$, em que foi mais baixa, comportamento semelhante ao observado para $P$. leiocarpa. Ambas espécies levaram longo tempo para germinação e emergência (Tabela 4). O nú- mero de sementes por fruto nesta espécie também é bastante variável, sendo que $48 \%$ deles tinham uma semente; $22 \%$ duas; $6,7 \%$ três sementes; $2,7 \%$ quatro sementes e $2 \%$ cinco ou seis sementes. Nenhuma semente foi encontrada em 18,6\% dos frutos examinados.

Dodonea viscosa teve taxa germinativa mais alta a $20^{\circ} \mathrm{C}$ no escuro (67\%), porém não diferindo dos outros tratamentos de temperatura, mas foi usada como a mais eficiente para a escolha do tratamento da emergência em areia (Tabela 4). Esta espécie germinou indiferentemente na luz ou escuro, sob diferentes temperaturas constantes entre $10^{\circ} \mathrm{e}$ $35^{\circ} \mathrm{C}$ ou ainda na alternância destas (Tabela $3)$. As sementes germinaram relativamente rápido - a partir de 3 dias (Tabela 4).

As sementes de Sida rhombifolia germinaram mais eficientemente a temperatura constante de $35^{\circ} \mathrm{C}$. Abaixo de $30^{\circ} \mathrm{C}$ a germinação foi inexpressiva, tanto na luz como na ausência desta. A alternância de temperaturas teve eficiência máxima quando no par foi incluída a temperatura de $35^{\circ} \mathrm{C}$ (Tabela 3 ). As sementes germinaram relativamente rápido (Tabela 4), especialmente a $35^{\circ} \mathrm{C}$.

\section{Discussão}

As sementes de $A$. gratissima são muito pequenas $(0,3 \mathrm{mg} / \mathrm{semente})$ e fotoblásticas positivas. É conhecido serem as sementes pequenas fotoblásticas. Ainda que a luz promova a germinação, esta foi baixa. A alternância de temperaturas é mais eficiente que a luz nesta promoção, o que pode indicar um mecanismo duplo envolvido na germinação - luz e temperatura - o que ocorre em sementes de várias espécies (Thompson, 1974). O teste de tetrazólio não foi realizado ao final da incubação (30 dias) com as sementes que não germinaram (cerca de 60\% de acordo com a tabela 3). Assim, não se tem condições de especular sobre as razões da germinabilidade relativa- 
Tabela 1 Conteúdo de umidade e peso de mil sementes de seis espécies lenhosas medicinais.

\begin{tabular}{lcccccc}
\hline & A,gratissima & B.forficata & C.sylvestris & D.viscosa & P.leiocarpa & S.rhombifolia \\
umidade \% & 5,0 & 14,0 & 9,5 & 8,8 & 9,5 & 7,6 \\
PMS. g. & 0,3 & 160,0 & 1,7 & 10,5 & 12,7 & 2,3 \\
\hline
\end{tabular}

Tabela 2 Germinação média de sementes de 6 espécies de plantas medicinais quanto a presença ou ausência de luz, substrato agar a $1 \%$.

\begin{tabular}{lllc}
\hline espécie & luz & escuro & fotoblastismo \\
\hline A .gratissima & $17,5(\mathrm{a})$ & $4,5(\mathrm{~b})$ & $*$ \\
B.forficata & $89,0(\mathrm{a})$ & $83,5(\mathrm{a})$ & 0 \\
C.sylvestris & $23,8(\mathrm{a})$ & $21,5(\mathrm{a})$ & 0 \\
D.viscosa & $59,5(\mathrm{a})$ & $57,8(\mathrm{a})$ & 0 \\
P.leiocarpa & $29,0(\mathrm{a})$ & $30,8(\mathrm{a})$ & 0 \\
S.rhombifolia & $22,0(\mathrm{a})$ & $20,8(\mathrm{a})$ & 0 \\
\hline
\end{tabular}

${ }^{*}=$ fotoblastismo positivo. Na linha letras iguais indicam diferenças não significativas a nível de $5 \%$. Teste Tukey

mente baixa de $A$. gratissima.

B. forficata apresentou germinabilidade acima de $80 \%$ em temperaturas constantes entre 15 e $30^{\circ} \mathrm{C}$, tanto na luz como no escuro. Estes dados contrastam com aqueles obtidos por Pereira (1992) onde a germinabilidade obtida foi bem mais baixa. Em outras coletas efetuadas em ambientes urbanos, obteve-se até $99 \%$ de sementes inviáveis por severo ataque de brocas e outros patógenos. Assim, para esta espécie, há que se ter especial cuidado sobre a sanidade das sementes. Sementes de B. forficata armazenadas em condições de laboratório $\left(22^{\circ} \mathrm{C}\right.$ \pm 2 ) ou em câmara fria a $5^{\circ} \mathrm{C}$, mantiveram-se com viabilidade acima de $90 \%$, por um ano (dados não mostrados).

O número e o tamanho das sementes podem variar em uma população de plantas de uma determinada espécie, e em cada planta, de fruto para fruto. Estes dados são importantes, pois podem estar diretamente relacionados com a germinação e o estabelecimento da plântula (Méndez, 1997). Em C. sylvestris o número de sementes por fruto variou de 1 a 6 . O percentual mais alto de germinação das sementes desta espécie foi de $47 \%$ a $25^{\circ} \mathrm{C}$, coincidentemente com o número de frutos com uma semente. $C$. sylvestris e P. leiocarpa são espécies nativas ocorrentes em matas no Rio Grande do Sul e a restrição de temperatura entre 20 e $25^{\circ} \mathrm{C}$ para a germinação, pode estar espelhando a situação de plantas de interior de mata onde, não havendo insolação direta plena, não há variações drásticas nas temperaturas diárias e sazonais.

Os frutos de D. viscosa foram coletados no final de janeiro, quando os tegumentos das sementes já estavam endurecidos, requerendo escarificação para germinar. As sementes escarificadas germinaram numa ampla faixa de temperatura na luz ou no escuro, ou ainda com alternância térmica (tabela 3). A época de coleta tem grande influência na germinabilidade das sementes. M.G.Rossoni (comunicação pessoal) encontrou que sementes escuras, coletas em novembro, podiam germinar até $93 \%$, enquanto as de cor verde não germinavam. No teste de tetrazólio, encontrou-se que as sementes escuras, não germinadas, eram viáveis. É citado na literatura (Burrows, 1995; Hussain et al., 1991) que a coloração dos tegumentos serve como indicativo da capacidade germinativa destas sementes, pois estão relacionados com a permeabilidade dos envoltórios das mesmas.

S. rhombifolia é conhecida como invasora de cultivos (Kissmann \& Groth, 1995). 
Tabela 3 Germinabilidade média (\%) de sementes entre as temperaturas constantes 20 a $35 \mathrm{C}$ ou alternantes, de 6 espécies de plantas lenhosas medicinais na presença ou ausência de luz, usando como substrato agar a $1 \%$.

\begin{tabular}{|c|c|c|c|c|c|c|}
\hline espécie & A .gratissima & B.forficata & C.sylvestris & D.viscosa & P.leiocarpa & S.rhombifolia \\
\hline \multicolumn{7}{|l|}{ Temperatura } \\
\hline \multicolumn{7}{|l|}{ Escuro } \\
\hline 10 & $1 \mathrm{de}$ & $0 \mathrm{c}$ & $0 \mathrm{~d}$ & $65 \mathrm{a}$ & $0 \mathrm{f}$ & $3 c$ \\
\hline 15 & $4 \mathrm{de}$ & $79 a b$ & $0 \mathrm{~d}$ & $61 \mathrm{a}$ & $0 \mathrm{f}$ & $2 \mathrm{c}$ \\
\hline 20 & $10 \mathrm{cde}$ & $86 a b$ & $37 a b$ & $67 \mathrm{a}$ & $57 \mathrm{abc}$ & $3 c$ \\
\hline 25 & 8 cde & 97 a & 39 a & $61 \mathrm{a}$ & $66 a$ & $2 \mathrm{c}$ \\
\hline 30 & $0 \mathrm{e}$ & $83 a b$ & $10 \mathrm{c}$ & 53 a & $0 \mathrm{f}$ & $24 b$ \\
\hline 35 & $0 \mathrm{e}$ & $68 \mathrm{~b}$ & $0 \mathrm{~d}$ & $50 a$ & $0 \mathrm{f}$ & $54 a$ \\
\hline \multicolumn{7}{|l|}{ luz } \\
\hline 20 & $24 \mathrm{abc}$ & $84 a b$ & $41 a$ & 56 a & $0 \mathrm{f}$ & $1 \mathrm{c}$ \\
\hline 25 & 16 bcde & $90 \mathrm{a}$ & $47 \mathrm{a}$ & $60 a$ & $62 a b$ & $1 \mathrm{c}$ \\
\hline 30 & $20 \mathrm{bcd}$ & 93 a & $7 \mathrm{~cd}$ & $61 \mathrm{a}$ & $0 \mathrm{f}$ & $26 \mathrm{~b}$ \\
\hline 35 & 10 cde & $89 a$ & $0 \mathrm{~d}$ & $61 \mathrm{a}$ & $0 \mathrm{f}$ & $60 \mathrm{a}$ \\
\hline \multicolumn{7}{|l|}{ alternância } \\
\hline 10 e 20 & 11 cde & $83 a b$ & 38 a & $66 a$ & 43 bcde & $2 c$ \\
\hline $15-25$ & $33 a b$ & $87 a b$ & 39 a & 49 a & 40 cde & $4 \mathrm{c}$ \\
\hline $20-30$ & $44 a$ & 94 a & $36 a b$ & $57 \mathrm{a}$ & $33 \mathrm{de}$ & $28 b$ \\
\hline $25-35$ & $37 \mathrm{ab}$ & $97 \mathrm{a}$ & $25 \mathrm{~b}$ & 55 a & $27 \mathrm{e}$ & 57 a \\
\hline $\mathrm{F}$ & 12.29 & 51.9 & 71.14 & 0.82 & 67.78 & 59.06 \\
\hline $\mathrm{P}$ & 0.00001 & 0.00001 & 0.00001 & 0.63 & 0.00001 & 0.00001 \\
\hline
\end{tabular}

Letras iguais nas colunas, diferenças não significativas ao nível de $5 \%$ (teste de Tukey).

Tabela 4 Germinação de sementes de 6 espécies medicinais lenhosas em ágar ou emergência em areia na temperatura ótima e avaliação da viabilidade das sementes ao final do experimento.

\begin{tabular}{|c|c|c|c|c|c|c|c|c|}
\hline \multirow[t]{2}{*}{ Espécie } & \multirow[t]{2}{*}{ temperatura } & \multicolumn{3}{|c|}{ germinação em ágar } & \multicolumn{3}{|c|}{ emergência em areia } & \multirow{2}{*}{$\begin{array}{r}\text { tetrazólio } \\
\text { positivo }\end{array}$} \\
\hline & & $\%$ & inicio & final ( dias) & $\%$ & inicial & final (dias) & \\
\hline A .gratissima & $20-30$ & 44 & 3 & 15 & 34 & 6 & 18 & * \\
\hline B.forficata & 25(escuro) & 97 & 3 & 24 & 82 & 6 & 27 & 89 \\
\hline C.sylvestris & 25 ( luz) & 47 & 12 & 33 & 32,5 & 21 & 45 & * \\
\hline D.viscosa & 20(escuro) & 67 & 3 & 15 & 58,5 & 3 & 18 & 63 \\
\hline P.leiocarpa & 25(escuro) & 66 & 18 & 27 & 43 & 21 & 33 & $52^{* *}$ \\
\hline S.rhombifolia & 35 (luz) & 60 & 3 & 9 & 39 & 6 & 15 & 75 \\
\hline
\end{tabular}

* Teste de tetrazólio não realizado.

** Quando a temperatura subiu a 30o C, ou mais, cerca de 1/3 da amostra manteve-se viável; nas temperaturas mais baixas, até o final do experimento ( 30 dias), havia cerca de 2/3 da amostra com sementes viáveis.

A unidade de dispersão constitui-se da semente e do pericarpo aderido, necessitando de escarificação para germinabilidade máxima (Cardoso, 1990). Este mesmo autor conclui que as sementes eram fotoblásticas negativas, resultados esses discrepantes com os verificados neste estudo. As sementes de S.rhombifolia foram fotoblásticas neutras e resultados semelhantes foram obtidos por outros autores (Smith et al., 1992). Estes autores mostraram que a germinabilidade foi mais alta a $35^{\circ} \mathrm{C}$, o que coincide com os resultados aqui obtidos e discordam daqueles de Cardoso (1990). Este autor armazenou por dois anos as sementes a $5^{\circ} \mathrm{C}$, ao contrário do presente trabalho onde não houve estocagem 
e daqueles obtidos por Smith et al.(1992), com apenas curta estocagem nesta temperatura. Também, não podem ser descartadas diferenças de material genético e/ou condições ambientais no periodo de formação do fruto e da semente, que se sabe pode alterar os requerimentos ecofisiológicos para germinação (Wulff, 1995).

\section{Agradecimentos}

À Dra. Maria Estefania A. Aquila, pela leitura crítica dos manuscritos e inúmeras sugestões à redação do texto. A CAPES, pela bolsa à S.G.T.Rosa e ao CNPq, por bolsa a A.G.Ferreira.

\section{Referências bibliográficas}

Alice, C.B.; Siqueira, N.C.S.; Mentz, 1.A.; Silva, G.A.A.B. \& José, K.F.D. 1995. Plantas medicinais de uso popular: atlas farmacognóstico. Ed. Universidade Luterana do Brasil, Canoas.

Bewley, J.D. \& Black, M. 1994. Seeds: Physiology of development and germination. Plenum Press, New York.

Brasil 1993. Regras para Análises de Sementes. Ministério da Agricultura e Reforma Agrária, Brasília.

Burrows, C.J. 1995. Germination behaviour of the seeds of six New Zealand woody plant species. New Zealand Journal of Botany. 33(3): 365377.

Cardoso, V.J.M. 1990. Germination studies on dispersal units of Sida rhombifolia L. Revista brasileria de Botânica 13(2): 83-88.

Deregibus, V.A.; Casal, J.J.; Jacobo, E.J.; Gibson, D.; Kaufman, M. \& Rodrigues, A.M. 1994. Evidence that heavy grazing may promote the germination of Lolium multiflorum seeds via phytochrome mediated perception of light red/far-red rations. Functional Ecology 8: 536- 542.

Dillenburg, C. R. 1978. A Tribo Psychotrieae (Rubiaceae) no Rio Grande do Sul. Dissertação de Mestrado. Universidade Federal do Rio Ghrande do Sul, Porto Alegre.

Elisabetsky, E.; Amador, T.A.; Leal, M.B.; Nunes, D.S. ; Carvalho, A.C.T. \& Verotta,L. 1997. Merging ethnopharmacology with chemotaxonomy: na approach to unveil biactive natural products. The case of Psychotria alkaloids as potential analgesics. Ciencia e Cultura 49(5/6): 378-409.

Farias, M.R. 1999. Avaliação da qualidade de materias primas Vegetais. Pp. 197-220. In, C.M.S.Simões; E.P.Schenkel; G.Gosmann; J.C.P.Mello; L.A.Mentz \& P.R.Petrovick. Farmacognosia: da planta ao medicamento. Ed.Universidade de Santa Catarina, Florianópolis.

Ferreira, A.G. 1977. Araucaria angustifolia(Bert.) O.Ktze.: germinação da semente e desenvolvimento da plântula. Tese de doutorado. Universidade de São Paulo, São Paulo.

Grabe, D.F. 1970. Tetrazolium testing handbook. Association of Official Seed Analyst. The tetrazolium Testing Commitee, Zurich.

Heydecker, W. 1977. Stress and seed germination: na agronomic view. Pp. 237-282. In: A.Khan (Ed.). The physiology and biochemistry of seed dormancy and germination. Elsevier, Amesterdam.

Hussain, F.; Shankat, S.; Ilahi, I. \& Qhreshi, M.Z. 1991. Note on the germination behavior of Dodonea viscosa (L.)Jacq. Scientific Khiber 4(1): 45-50.

Kendrick, R. \& Kronenberg, G. H. M. 1994. Photomorphogenesis in Plants. Martinus Nijhoff, Dordrecht.

Kissmann, K. \& Groth, D. 1995. Plantas Infestantes e Nocivas v. 3. Basf, São Paulo.

Labouriau, L.G. 1983. A germinação das sementes. Secretaria-Geral da organização dos Estados Americanos. Washington.

Labouriau, L.G. \& Costa, J.A.F. 1976. Objetivos e instalações básicas de um laboratório de fisiologia vegetal. Academia Brasileira de Ciências, Rio de Janeiro.

Lopes, A.M.V. 1997. Plantas usadas na medicina popular do Rio Grande do Sul. Ed. Incograph, Santa Maria.

Lorenzi, H. 1992. Árvores brasileiras: manual de identificação e cultivo de plantas arbóreas nativas do Brasil. Ed. Plantarum, Nova Odessa.

Méndez, M. 1997. Sources of variation in seed mass in Arum italicum. International Journal of Plant Science 158(3): 298-305.

Pereira, T. S. 1992. Germinação de sementes de Bauhinia forficata Link (Leguminosae, Caesalpinoideae). Revista brasileira de Sementes 14(1): 77- 82 . 
Rao, K.S. \& Mishra, S.H. 1997. Anti-inflammatory and hepatoprotective activities of Sida rhombifolia L. Indian Journal of Pharmacology 29(2): 110-116.

Reis, A.; Zambonim, R.M. \& Nakazone, E. 1998. Recuperação de áreas florestais degradadas utilizando a sucessão e as interações planta-animal. Cadernos da Biosfera da mata Atlântica 14. São Paulo.

Rosa, S.G.T. \& Ferreira, A.G. 1999. Germination of medicinal plant: Smilax campestris Griseb (Salsaparrilha). Acta Horticulturae 502: 105111.

Rosa, S.G.T. \& Barros, I.B.I. 1999. Behaviour of Maytenus ilicifolia seeds in different periods and storage conditions. Acta Horticulturae 502: 249254.

Rosa, S.G.T. \& Ferreira, A.G. 1998. Germinação de sementes de espécies medicinais do Rio Grande do Sul: Bromelia antiacantha Bert.; Cuphea carthagenensis (Jacq.) Macbride e Talinum patens (Jacq.)Willdenow. Acta Botanica Brasilica 12(3): 515-522.

Rosa,S.G.T. 2000. Germinação de sementes de espécies medicinais da flora do Rio Grande do Sul. Tese de Doutorado. Universidade Federal do Rio Grande do Sul, Porto Alegre.

Sanchotene, M.C.C. 1989. Frutíferas nativas úteis à fauna na arborização urbana. Sagra, Porto Alegre.

Scopel,A.L.; Ballaré,C.L. \& Radosevich,S.R. 1994. Photo stimulation of seed germination during soil tillage. New Phytologist 126: 145-152.

Simões, C.M.O.; Mentz, L.A.; Schenkel, E.P.; Irgang,B.E. 1986. Plantas da medicina popular no Rio Grande do Sul. Ed. Universidade/UFRGS, Porto Alegre.

Smith, C. A. ; Shaw, D.R. \& Newsom, L. J. 1992. Arrow leaf sida (Sida rhombifolia) and prickly sida (Sida spinosa): germination and emergence. Weed Research 32: 103- 109.

Sokal,R. R. \& Rohlf,F.J. 1981. Biometry. W.H.Freeman, San Francisco.

Thompson, K. \& Grime, J.P. 1983. A comparative study of germination responses to diurnallyfluctuating temperatures. Journal of Applied Ecology 20: 141-156.

Thompson, P.A. 1974. Effects of fluctuating temperatures on germination. Journal of Experimental Botany 25(84): 164-175.

Toole,V.K., 1973. Effects of light, temperature and their interactions on the germination of seeds .
Seed Science \& Technology 1: 339-396.

Wulff,R.D. 1995. Environmental maternal effects on seed quality and germination. Pp. 491-505. In J.Kigel \& G.Galili. Seed Development and Germination. Marcel Decker, New York. 